\title{
Derivation of a pitch angle value for the motorcycle
}

\author{
Mateusz Lazarek, Juliusz Grabski, and Przemyslaw Perlikowski ${ }^{\mathrm{a}}$ \\ Division of Dynamics, Lodz University of Technology, Stefanowskiego 1/15, \\ 90-924 Lodz, Poland
}

Received 9 December 2019 / Accepted 8 June 2020

Published online 28 September 2020

\begin{abstract}
In this paper we consider a new, highly precise model of the kinematics of the motorcycle. We present an iterative method which let us calculate a proper pitch angle value and position of the front wheel tyre-ground contact point. We take into account system with and without the lateral flexibility of the front forks. We reveal different behaviour during maneuver of system with finite lateral stiffness compared to rigid one. We proof the dependence between the capsize mode and the pitching behaviour. We show that presented algorithm can be applied to the electronic control units protecting from wheelie behaviour.
\end{abstract}

\section{Introduction}

Motorcycle, like all two wheeled vehicles, in a stationary situations always has two points of support. It can change during aggressive riding when wheelie (front wheel raised off the ground during ride) or stoppie (rear wheel raised off the ground during ride) can occur. However, in most cases two points support is retained. Basic steering action like rotation of steering bars results in leaning motion of the motorcycle which causes the vertical movement of the steering head and in consequence all attached parts. In real word, the gravity force acting on the motorcycle ensures two points contact naturally. Nevertheless in mathematical model, due to complex geometry of the motorcycle, the specific constrains should be taken into account to keep tyreground contact of both wheels.

Importance of involving pitching motion into mathematical model, thereby preserving the two-points contact is caused by tyre modelling [1]. Generally speaking, without including the pitching motion in the model of a motorcycle it is impossible to obtain the proper tyre-ground interactions and maintain two contact points. The precise definition of contact points' constrains and tyre model has been investigated through years. The basic approach is a string model introduced by Koenen [2]. Tyre string model, in its basic form, models the tyre as the string attached to the circumference of the rigid disk by means of an infinite number of lateral springs. Further motorcycle tyre models use the Magic Formula, where the overturning moment is calculated separately and than added to the model [3]. The most advanced approach

\footnotetext{
a e-mail: przemyslaw.perlikowski@p.lodz.pl
} 
includes the tyre forces and the torques acting on the actual contact point finally gives very good correspondence to the measurements of forces in both steady state and transient tire behaviour [4].

We propose an iteration algorithm which enables calculation of pitch angle value by computation of the precise position of the tyre-ground contact points. Hence the value of pitch angle is corrected until the contact points are reached with assumed precision. We introduce plots depicting changes in tyre-ground contact points and pitch angle value for different values of roll and steering angles. One can find different, less accurate approach of derivation proper pitch angle value of rear frame [5]. It has been used for calculations in [6,7]. The pitch motion can be neglected if one wants only to investigate the stability of straight motion [2]. Nevertheless implementation of suspension and/or large values of steering angle increase the importance of considering of the pitching motion. The necessity of full representation of motorcycle motion has been highlighted by Honda and implied in first motorcycle simulator [8] which has been further developed by Nehaoua et al. [9].

Another part of mechanical structure of the motorcycle which has to be taken into account in precise model of tyre-ground interactions is flexibility of the bodies. Stiffness of the frame influences the response of the motorcycle as well as it changes values of parameters (position of wheel base, rake angle, trial, ect.). Necessity of considering lateral flexibility has been studied in the early 80 s of the nineteenth century $[10,11]$. We extend the basic motorcycle model by addition of the flexible forks. This flexibility is taken into account because lateral forks stiffness is relatively small compared to other frame's stiffnesses [12].

The paper is organized as follows. In Section 2 we describe the model of the motorcycle. Section 3 is devoted to kinematic relations and algorithm which let us calculate the proper pitch angle. In Section 4 we show the numerical results. Finally, in Section 5 the conclusions are drawn.

\section{Model of the motorcycle}

In our study the motorcycle model consists of five bodies: rear frame, upper front frame, lower front frame, rear and front wheel [2]. The general scheme of the model is presented in Figure 1. We mark aforementioned bodies as follows: orange -1 (rear frame), blue - 2 (upper front frame), grey - 3 (lower front frame), pink - 4 (rear wheel) and pink - 5 (front wheel). We need to select set of characteristic points to determinate the relation between bodies during motion of motorcycle: Points $C_{1}$ and $C_{2}$ are front and rear wheel contact points, $W_{1}$ and $W_{2}$ are front and rear wheel points laying at intersection of wheel axis and wheel longitudinal plane of symmetry, $F$ is a steering head, $J$ is a point at intersection of front upper frame longitudinal plane of symmetry and front frame torsion axis and $K$ is a point at the end of vertical part of front assembly.

We assume that all bodies are rigid and wheels are described as thin discs, while points $J$ and $F$ coincide. Point $C_{2}$ is a centre of main coordinate system which is moving with motorcycle structure. Position of point $C_{2}$ is at the intersection of three planes: $P_{1}$ (the vertical transverse plane containing the rear wheel pivot), $P_{2}$ (the longitudinal plane of symmetry), and $P_{g}$ (the ground plane) as shown in Figure 1a. Reference axis directions are introduced according to SAE vehicle dynamics standards [13]. We consider the roll angle $-\varphi$, the pitch angle $-\theta$, the steering head angle $-\epsilon$, the steering angle $-\delta$ and the front frame torsion angle $-\beta$. The values of angles and lengths that characterize the geometry allow for unambiguous determination of the position of the motorcycle.

In the real situation the rotation of the handlebars causes simultaneous roll motion of the motorcycle. This property is used in countersteering technique which allows 


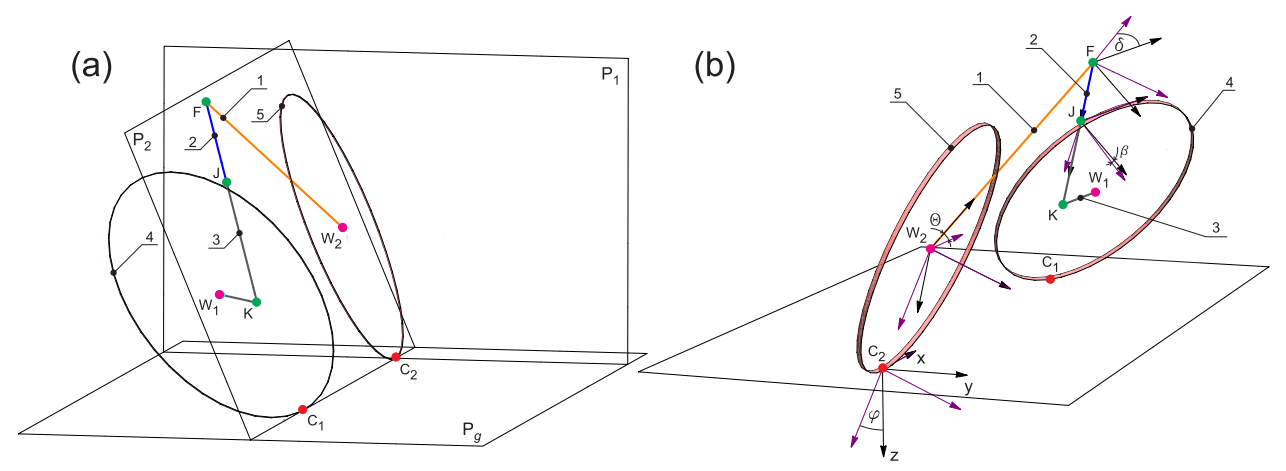

Fig. 1. Motorcycle model in two different views. In panel (a) we present planes $P_{1}, P_{2}$ and $P_{g}$, while in panel (b) we show the systems of coordinates and angles used in presented model.

(a)

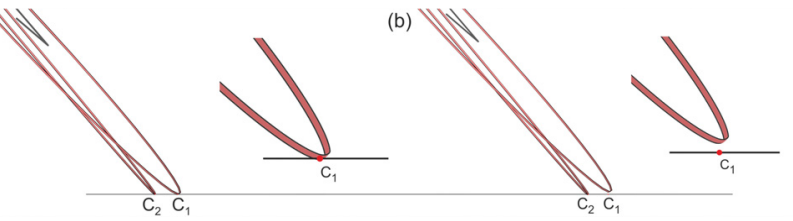

Fig. 2. The difference between models with correct (a) and incorrect (b) front tyre-ground constrain.

to minimize the human effort in cornering maneuver. In case of right corner countersteering technique demands counter-clockwise rotation of the handlebar which results in increase of roll angle $\varphi$. Then the rider has to rotate the handlebar in the clockwise direction in order to receive cornering trim state. Last stage is exit of the corner with counter-clockwise rotation of the handlebars and finally return to straight running state (for details see [14]). Note that, even typical parameters' configuration referring to stage of coming in or out of a corner with countersteering action can lead to detachment of the wheel from the ground plane. To properly model this behaviour we need to calculate the correct value of pitch angle. In the case of straight motion of motorbike with steering angle equals to zero, the pitch angle can be found based on basic geometrical relations. Unfortunately, even the small change in the input parameters (perturbation from straight motion) makes this calculations more complex mainly due to multiple projections. To simplify analysis one can use a transformation matrices in calculations. Disturbing effects from changes in the roll angle $\varphi$, the steering angle $\delta$ and the front frame torsion angle $\beta$ acting on the front tyre-ground constraints are shown in Figure 2. The incorrect model of tyre ground interaction may lead to detachment of the front wheel. The correct result is shown in Figure 2a, while the incorrect in Figure 2b. The examples are calculated based on our model of motorcycle kinematic.

\section{Kinematic relations}

\subsection{Main relations}

In this section we derive the exact position of the characteristics motorcycle's points using the theory of transformation matrices [15]. Position of those points can be 
obtained by following transformation:

$$
p^{0}=\mathbf{A} p^{S}
$$

where $p^{0}$ is a position of the body in global arbitrary coordinate system $0, \mathbf{A}$ corresponds to transformation from arbitrary local system $S$ to system 0 and $p^{S}$ is a position of desired point of body in coordinate system fixed with body. This scheme can be applied when required angles are known a priori. For motorcycle geometry one have to derive the position and orientation of the front assembly. Let us introduce an auxiliary unit vector which is directed along the wheel axis and is defined in the global coordinate system:

$$
j=\mathbf{A}^{\varphi} \mathbf{A}^{\theta} \mathbf{A}^{\epsilon} \mathbf{A}^{\delta} \mathbf{A}^{\beta} e_{2}
$$

where $e_{1,2,3}$ is a standard basis for unit vectors arrangement of the global coordinate system. Rotation matrices are given as follows:

$$
\begin{aligned}
& \mathbf{A}^{\varphi}=\left[\begin{array}{ccc}
1 & 0 & 0 \\
0 & \cos \varphi & -\sin \varphi \\
0 & \sin \varphi & \cos \varphi
\end{array}\right] \quad \mathbf{A}^{\theta}=\left[\begin{array}{ccc}
\cos \theta & 0 & \sin \theta \\
0 & 1 & 0 \\
-\sin \theta & 0 & \cos \theta
\end{array}\right] \\
& \mathbf{A}^{\epsilon}=\left[\begin{array}{ccc}
\cos \epsilon & 0 & \sin \epsilon \\
0 & 1 & 0 \\
-\sin \epsilon & 0 & \cos \epsilon
\end{array}\right] \quad \mathbf{A}^{\delta}=\left[\begin{array}{ccc}
\cos \delta & -\sin \delta & 0 \\
\sin \delta & \cos \delta & 0 \\
0 & 0 & 1
\end{array}\right] \\
& \mathbf{A}^{\beta}=\left[\begin{array}{ccc}
1 & 0 & 0 \\
0 & \cos \beta & -\sin \beta \\
0 & \sin \beta & \cos \beta
\end{array}\right] \quad \mathbf{A}^{\delta^{\prime}}=\left[\begin{array}{ccc}
\cos \delta^{\prime} & -\sin \delta^{\prime} & 0 \\
\sin \delta^{\prime} & \cos \delta^{\prime} & 0 \\
0 & 0 & 1
\end{array}\right] \\
& \mathbf{A}^{\gamma}=\left[\begin{array}{ccc}
1 & 0 & 0 \\
0 & \cos \gamma & \sin \gamma \\
0 & -\sin \gamma & \cos \gamma
\end{array}\right] \quad \mathbf{A}^{\theta_{f}}=\left[\begin{array}{ccc}
\cos \theta_{f} & 0 & \sin \theta_{f} \\
0 & 1 & 0 \\
-\sin \theta_{f} & 0 & \cos \theta_{f}
\end{array}\right] \text {. }
\end{aligned}
$$

Using equation (2) the front wheel roll angle and the effective steering angle can be calculated as follows:

$$
\begin{gathered}
\gamma=\arcsin \left(j \cdot e_{3}\right) \\
\delta^{\prime}=\arctan \left(-j \cdot e_{1} / j \cdot e_{2}\right) .
\end{gathered}
$$

Different strategy is performed to calculate the front pitch angle $\theta_{f}$. In that case, we use the law of cosines to find the value of an angle laying between two vectors, namely $K \bar{W}_{1}$ and $W_{1}^{-} C_{1}$. Resulting value should be subtracted from the $\pi / 2 \mathrm{rad}$. Final formula for the front pitch angle reads:

$$
\theta_{f}=\frac{\pi}{2}-\arccos \frac{K \bar{W}_{1} \cdot W_{1}^{-} C_{1}}{\left\|K \overline{W_{1}}\right\|\left\|W_{1}^{-} C_{1}\right\|} .
$$

Parts of this methodology is described in [2] - derivation of the front wheel constraints, roll angle, the effective steering angle and derivation of the front wheel radius vector. Sequence of locating the specific point position is critical issue. For analysis 
which starts at $C_{2}$ point - central point of the rear frame in global system of coordinates, the rotation order should be as listed in Section 2. First rotation concerns rolling motion with the value of the roll angle $\varphi$, then the motorcycle is rotated about its rear wheel pivot by the pitch angle value $\theta$. Next steps refer to rotations by the steering head angle $\epsilon$, the steering angle $\delta$ and the front frame torsion angle $\beta$. Contrary when assuming $C_{1}$ - central point of the front frame global system of coordinates as the starting point one should follow different folding scheme. At first wheel should be rotated about vertical axis by the effective steering angle value $\delta^{\prime}$. Then one has to lean wheel with the front wheel roll angle $\gamma$ and the front pitch angle $\theta_{f}$. Geometry of the front frame assembly also should be taken into account. This can be obtained by introducing an angle between steering axis and offset of wheel spindle from steering axis. In presented model position of construction points is given by parameters listed in Table 1 . Value of front frame torsion angle is calculated based on forks lateral stiffness $K_{f f}$ [12], which vary in range from 0.07 to $0.18 \mathrm{kN} / \mathrm{mm}$. We assume that typical weight of the motorcycle with rider is equal to $300 \mathrm{~kg}$ and is distributed uniformly to front and rear wheel of motorcycle. We also assume that the lateral acting force is expressed as: $Q_{l}=Q \cos \left(\frac{\pi}{2}-\varphi\right)$. After calculation of forks deflection with use of equation: $x_{f}=Q_{l} / K_{f f}$ we are able to get the value of front frame torsion angle $\beta$.

\subsection{Algorithm}

In order to get the proper value of the pitch angle one has to notice that the position of an arbitrary construction point can be expressed in different global coordinate systems. Central point of the first, global system is fixed in $C_{2}$ point which is the tyre-ground contact position of the front tyre. The second one, coincides with $C_{1}$, where the front wheel touches the ground. Possibility of definition of the arbitrary point of the structure location in two aforementioned coordinate systems is presented onward.

Collection of this data becomes useful for comparison purpose only after expressing them in one major coordinate system. That means that point calculated in $C_{1}$ system must be transferred to $C_{2}$ using previously calculated front wheel radius vector. Almost all points' positions computed with presented method can be compared with assumed feedback error value. Different pairs of points need specific algorithms which affect system complexity. The choice of correct pair of points have significant influence on achieving precise results. In this work the comparison between positions of the steering head has been used. The flow chart of algorithm used for calculation is shown in Figure 3.

This short introduction let us present a description of all operations of the proposed algorithm. At the beginning we introduce parameters of the vehicle. The next step is the updating of values (in first run the values stay unchanged) which have been calculated in the previous loop run. After assignation of values the calculation of construction points in two coordinate systems is executed. Since then position of main construction points become stored in predestined variables. As aforementioned the unification of coordinate systems is needed and we include this in this step. The next action is calculation of the difference between positions of points $F_{f}$ and $F_{r}$. Those points are placed at steering head but they are described in front and rear system of axes respectively (our goal is to obtain the same position of both points in one of the global coordinate systems). The error value is derived based on the law of cosines. Note that points $W_{2}, F_{f}$ and $F_{r}$ create triangle which vertices allows direct implementation of trigonometry law. This triangle consists the searched angle $\varangle F_{f} W_{2} F_{r}$. Obtained value is compared with the original value from first step or previous one in second and subsequent iterations. Finally if positions of points $F_{r}$ and $F_{f}$ have the 


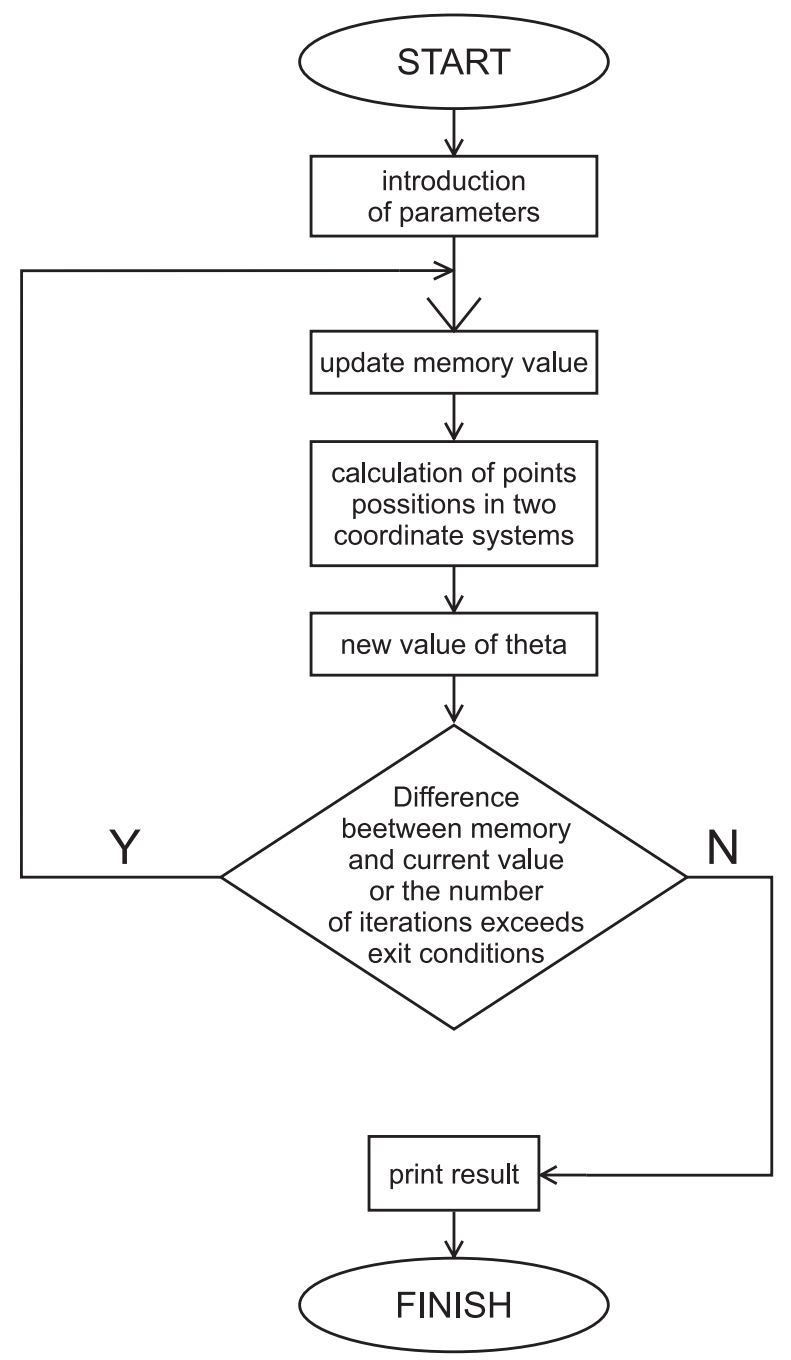

Fig. 3. The main algorithm loop. The iterative method calculates the proper pitch angle value and position of the front wheel tyre-ground contact point based on the error value (difference between positions of points $F_{f}$ and $F_{r}$ ).

same values with permissible error in one global coordinate system the exit condition is fulfilled. The exit condition allows control of solution accuracy which can be adjusted to specific purposes, e.g., motorcycle anti-wheelie system. Of course several extensions can be added to presented algorithm in straightforward manner such as more precise tyre model or front and rear suspension. This algorithm has been used as a core of program and it is able to demonstrate results dependent from changes in the roll and steering angles. Presented model is implemented in Mathematica [16].

\section{Results}

In this section the influence of changing of the roll $\varphi$ and the steering $\gamma$ angles on the value of of the pitch angle is presented. The pitch angle value for the straight 

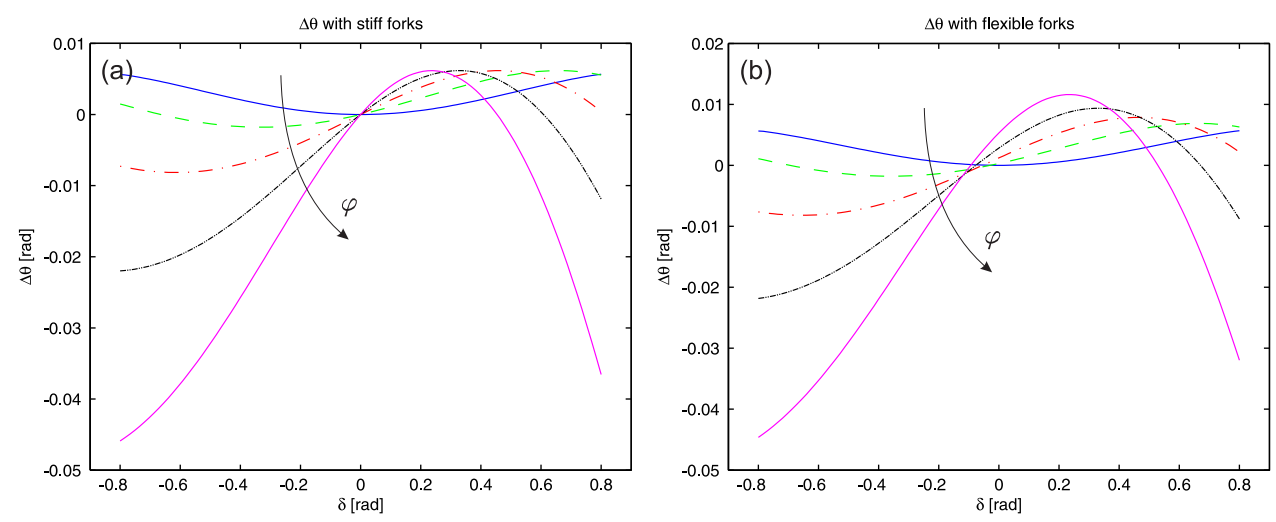

Fig. 4. Change of the pitch angle value under changes of the steering angle for $\beta=0 \mathrm{rad}$ (a) and with variable front frame torsion angle $\beta$ (b). Colours of lines refer to: $\varphi=0$ rad - solid blue; $\varphi=0.18 \mathrm{rad}$ - dashed green; $\varphi=0.36 \mathrm{rad}-$ dash-dotted red; $\varphi=0.54 \mathrm{rad}-$ dash-double dotted black and $\varphi=0.72 \mathrm{rad}-$ three dash-dotted magenta.

running is equal to $\theta_{0}=0.484 \mathrm{rad}$ and in this paper we show the changes of the pitch angle value in respect to this value $\theta=\theta_{0}+\Delta \theta$. Due to the symmetry the results for positive and negative values of roll angle $\varphi$ of the motorcycle are identical, hence we show just positive part. All results have been verified using the three dimensional motorcycle model coded in Mathematica software. At the beginning we discuss results for the motorcycle with the stiff front frame assembly where torsion is impossible. The steering angle varies from $-0.8 \mathrm{rad}$ to $0.8 \mathrm{rad}$ with step equal to $0.016 \mathrm{rad}$. The chosen maximum and minimum values correspond to accessible range of steering angle. In practice the full steering angle range is restricted by the geometry of the front steering system and stops on the triple clamp. In Figure 4a one can find characteristic (solid blue line) for motorcycle in vertical position which shows growth of the pitch angle value during clockwise and counter-clockwise rotation of the steering system. The chart is symmetric in respect to zero value of the steering angle $\delta$, extreme values are equal to 0 and $0.0057 \mathrm{rad}$. Increase of the roll angle $\varphi$ changes the shape and brakes the symmetry which is clearly visible for all four chosen values of $\varphi$, i.e. $\varphi=0.18 \mathrm{rad}$ (dashed green line), $\varphi=0.36 \mathrm{rad}$ (dash-dotted red line), $\varphi=0.54 \mathrm{rad}$ (dash-double dotted black line) and $\varphi=0.72 \mathrm{rad}$ (three dash-dotted magenta line). During the clockwise rotation of the front wheel, change of the pitch angle value increases up to $0.0062 \mathrm{rad}$ which is the maximum value among all asymmetric cases. Further increase of the roll angle changes the value of the steering angle where the monotonicity change is observed. Increase of the steering angle $\delta$ during the counter-clockwise rotation also affects the curvature of line. For $\varphi=0.18 \mathrm{rad}$ and $\varphi=0.36 \mathrm{rad}$ we observe the change of the slope for $\delta=0.66 \mathrm{rad}$ and $\delta=0.46 \mathrm{rad}$ with minimum values of change of the pitch angle $\Delta \theta=-0.0081 \mathrm{rad}$ and $\Delta \theta=-0.0018 \mathrm{rad}$ respectively. In case of $\varphi=0.54 \mathrm{rad}$ and $\varphi=0.72 \mathrm{rad}$ there is no change of the slope and the minimum values of change of the pitch angle at the end of considered range of steering angle $\delta$ are $\Delta \theta=-0.0220 \mathrm{rad}$ and $\Delta \theta=-0.0459 \mathrm{rad}$ respectively.

The results calculated for motorcycle with flexible forks are presented in Figure $4 \mathrm{~b}$. For the roll angle equals to zero the obtained values of change of the pitch angles are exactly the same as for the motorcycle with the stiff forks and we mark them as previously by blue lines. During computation of the pitch angle for the flexible forks we take into account the change of the wheel base which affect the front frame torsion angle $\beta$. Similarly as in previous case, we consider four non zero values of steering angle, i.e. $\varphi=0.18 \mathrm{rad}$ (dashed green line), $\varphi=0.36 \mathrm{rad}$ (dash-dotted red 

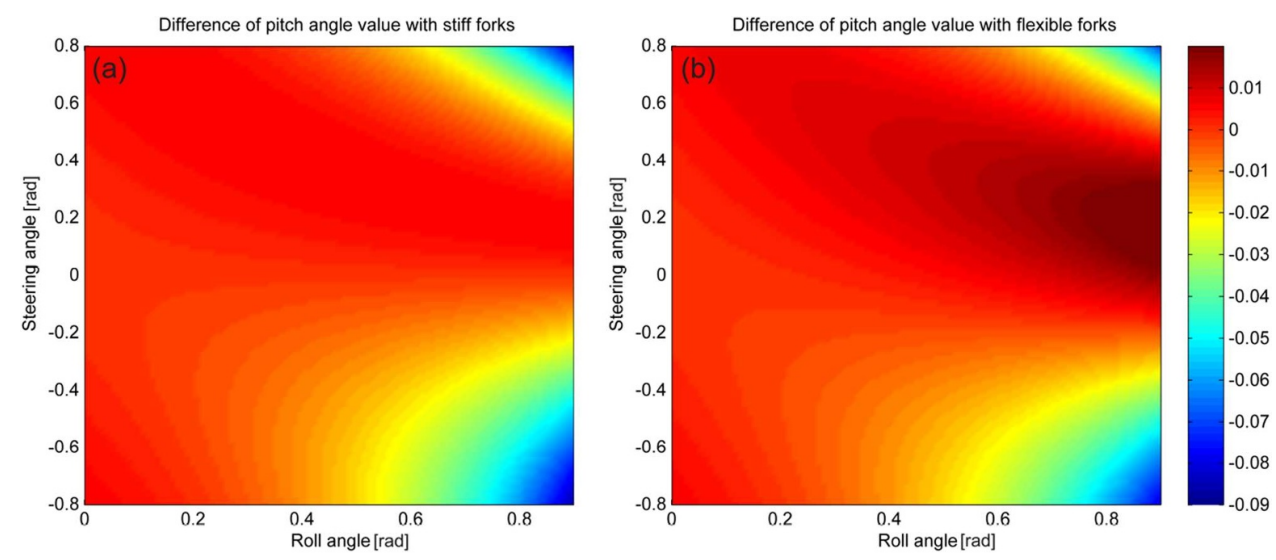

Fig. 5. Change of pitch angle value as a function of the roll and steering angles (a) $\beta=0$ and (b) $\beta \neq 0$.

line), $\varphi=0.54 \mathrm{rad}$ (dash-double dotted black line) and $\varphi=0.72 \mathrm{rad}$ (three dashdotted magenta line). As we can see with increasing of the roll angle $\varphi$ the steering angle $\delta$ for which the change of the pitch angle $\Delta \theta$ is equal to zero is not in the same point and it moves towards the negative values of the steering angle $\delta$. The values of change of the pitch angle value $\Delta \theta$ are strongly affected and we observe that its maximum values are even twice larger than for the stiff forks and occur for smaller steering angle $\delta$. The maximum values of change of the pitch angle are as follow for $\varphi=0.18 \mathrm{rad}\left(\Delta \theta_{\max }=0.0069 \mathrm{rad}\right), \varphi=0.36 \mathrm{rad}\left(\Delta \theta_{\max }=0.0079 \mathrm{rad}\right), \varphi=0.54$ $\operatorname{rad}\left(\Delta \theta_{\max }=0.0094 \mathrm{rad}\right)$ and $\varphi=0.72 \mathrm{rad}\left(\Delta \theta_{\max }=0.0116 \mathrm{rad}\right)$. The same change is observed for second intersection with $\Delta \theta=0 \mathrm{rad}$, but now it occurs for larger values of the steering angle $\delta$, hence the range of the positive pitch angle is wider. To show this effect we compare the positive pitch angle range for the roll angle $\varphi=0.72 \mathrm{rad}$, e.g., for the stiff forks zero crossing points are for $0 \mathrm{rad}$ and $0.34 \mathrm{rad}$ while for flexible forks for $-0.18 \mathrm{rad}$ and $0.5 \mathrm{rad}$. Nevertheless, the shape of lines stay unchanged and their monotonic is preserved. The minimum values of change of the pitch angle $\Delta \theta$ in the considered range of steering angle $\delta$ are nearly the same as for the stiff forks: $\varphi=0.18 \mathrm{rad}\left(\Delta \theta_{\min }=-0.0018 \mathrm{rad}\right), \varphi=0.36 \mathrm{rad}\left(\Delta \theta_{\min }=-0.0082 \mathrm{rad}\right), \varphi=0.54$ $\operatorname{rad}\left(\Delta \theta_{\min }=-0.0218 \mathrm{rad}\right)$ and $\varphi=0.72 \mathrm{rad}\left(\Delta \theta_{\min }=-0.0446 \mathrm{rad}\right)$.

To give more details we present the difference between system with and without finite lateral stiffness of forks in two parameters plots in Figure 5.

Plots show change of the pitch angle values as a function of steering angle $\varphi$ (vertical axis) and roll angle $\delta$ (horizontal axis). We plot them in the following ranges of roll $\varphi \in\langle 0,0.9\rangle \mathrm{rad}$ and steering $\delta \in\langle-0.8,0.8\rangle \mathrm{rad}$ angles. First of all one can see that the maximum value of the pitch angle is larger for system with the deformable forks. Extrema values are equal to $\Delta \theta_{\max }=0.006 \mathrm{rad}, \Delta \theta_{\min }=-0.086 \mathrm{rad}$ and $\Delta \theta_{\max }=0.024 \mathrm{rad}, \Delta \theta_{\min }=-0.08 \mathrm{rad}$ for the stiff and flexible forks respectively. In case of system with the finite lateral stiffness of forks the increase of roll angle $\varphi$ results in the noticeable shift of the maximum pitch angle value towards smaller steering angle $\delta$ values. Minima are reached for similar values of both parameters but system with the stiff forks poses larger range where the pitch angle value is lower then $-0.02 \mathrm{rad}$. It causes that the slope of characteristics in case of the flexible forks is more steep, hence the changes of the pitch angle occurs more rapidly.

Another important issue is an influence of change of the pitch angle values on the dynamical behaviour of the motorcycle. The motorcycle has three main modes of vibrations: the capsize, the weave and the wobble modes. Recalling briefly, the capsize 


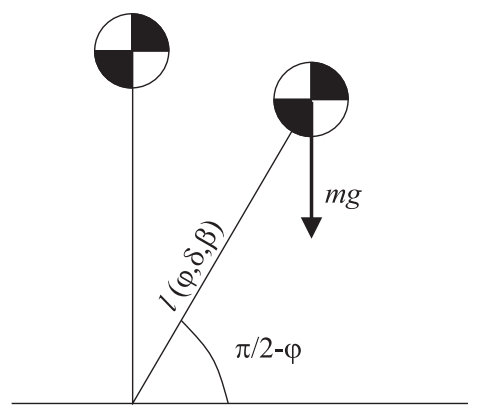

Fig. 6. Rear view on the motorcycle in the vertical position and rolled of $\varphi$.

mode (falling over) occurs at low-speeds of motorcycle and it is easy to control it by the rider. The weave is often compared to the motion of the fish tail. The last one is the wobble mode which is responsible for oscillations of the front assembly relative to the main frame of the vehicle $[12,17]$. Most of articles focus on the wobbling and waving modes due to the inability to control them by the rider. However dynamics concerned with capsize mode is interesting mainly because of the motorcycle kinematics. Short overview of dynamics of capsize mode can be found in $[18,19]$.

The equation of rolling motion can be described as follow:

$$
I \ddot{\varphi}=m g l(\varphi, \delta, \beta) \cos \left(\frac{\pi}{2}-\varphi\right)
$$

where $\varphi$ is the roll angle, $I$ is the total moment of inertia about axis defined by $C_{1}$ and $C_{2}$ points when the motorcycle is in the vertical position, $m$ is the total mass of motorcycle and $l(\varphi, \delta, \beta) \cos \left(\frac{\pi}{2}-\varphi\right)$ is the moment arm. Geometrical decryption of this kind of motion is shown in Figure 6.

The hypothesis introduced in [19] assumes that the value of $l(\varphi, \delta, \beta)$ is an increasing function of the moment arm during the capsize action. All results presented in Figures 4 and 5 confirm aforementioned hypothesis. Moreover pitching motion can excite vibrations along the motorcycle's plane of symmetry, especially in the case of wobbling. Where high frequency oscillations of the steering system are harder to damp out because of complex motion.

The pitch angle is not the only variable which changes its value due to variation of the configuration of the motorcycle. We also observe its strong influence on the position of the type-ground contact point. In Figures 7 and 8 we show the change of position of the front wheel contact point in $x$ and $y$ components of the rear frame global system of coordinates (see Fig. 1b) as a function of steering $\delta$ and roll angles $\varphi$.

In Figure 7 we show the results for the stiff framed motorcycle. One can see that the difference between the clockwise and the counter-clockwise direction of varying of the steering angle $\delta$ is significant for both $x$ and $y$ coordinates. Changes of $x$ and $y$ components of the stiff framed motorcycle influence the wheel's load and the cornering ability of the motorcycle. Lateral move of the front wheel contact point shows two zero crossing points for each roll angle (except $\varphi=0$ rad). In real maneuver using the countersteering action the contact point can cross the plane of the motorcycle symmetry more than two times during enter and exit of the corner. For zero value of the roll angle $\varphi$ the charts are symmetric with respect to zero value of the steering angle $\delta$. In case of $x$ components extreme values are equal to $1.1047 \mathrm{~m}$ and $1.0565 \mathrm{~m}$. We observe similar amplitude for $y$ components which extreme values are $\pm 0.0206 \mathrm{~m}$. Increase of the roll angle $\varphi$ changes the shape and breaks the symmetry which is 

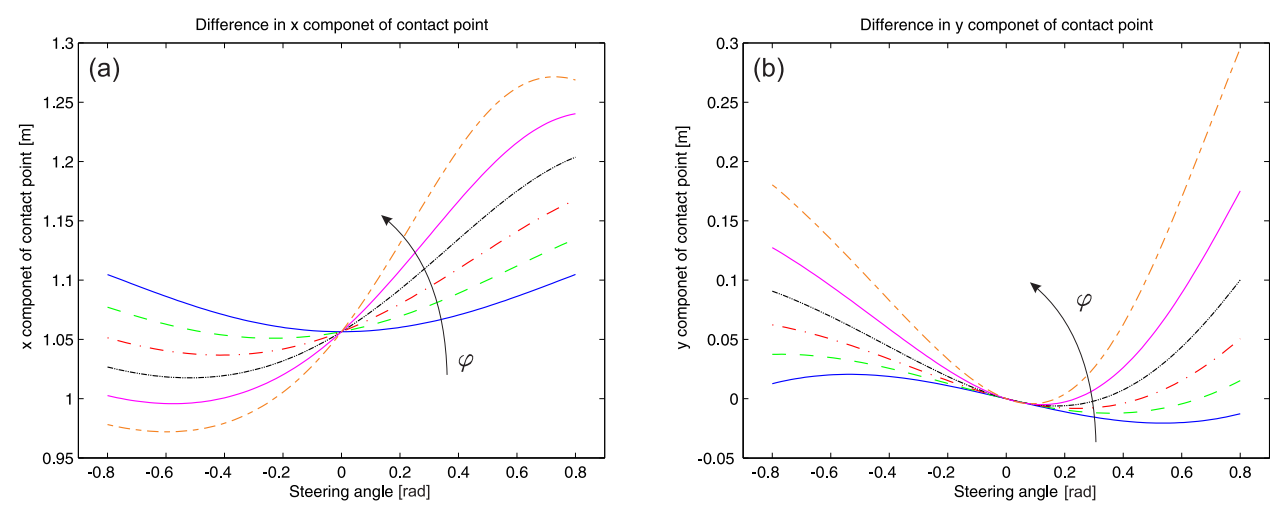

Fig. 7. Change of front wheel tyre-ground contact position under changes in roll and steering angles for motorcycle with the stiff forks. Colours of lines refer to: $\varphi=0 \mathrm{rad}-$ solid blue; $\varphi=0.18 \mathrm{rad}-$ dashed green; $\varphi=0.36 \mathrm{rad}-$ dash-dotted red; $\varphi=0.54 \mathrm{rad}-$ dash-double dotted black; $\varphi=0.72 \mathrm{rad}$ - three dash-dotted magenta $\varphi=0.9 \mathrm{rad}-$ long and short dashed orange.
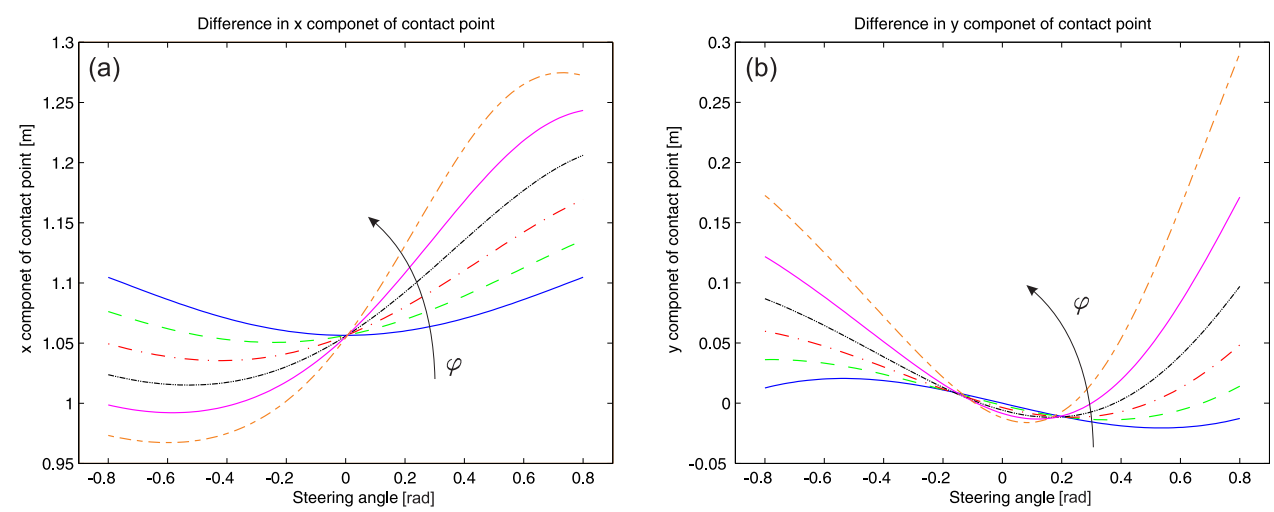

Fig. 8. Change of front wheel tyre-ground contact position under changes in roll and steering angles for motorcycle with the flexible forks. Colours of lines refer to: $\varphi=0 \mathrm{rad}-$ solid blue; $\varphi=0.18 \mathrm{rad}$ - dashed green; $\varphi=0.36 \mathrm{rad}$ - dash-dotted red; $\varphi=0.54 \mathrm{rad}-$ dash-double dotted black; $\varphi=0.72 \mathrm{rad}$ - three dash-dotted magenta $\varphi=0.9 \mathrm{rad}-$ long and short dashed orange.

clearly visible for all four chosen values of $\varphi$. Simultaneous increase of the roll angle and clockwise rotation (change of the steering angle $\delta$ ) of handlebars changes and increases the maximum value of $x$ component. The general behaviour has exception for large roll angles $\varphi$ which is unheard of reality. Similar effects can be observed in case of $y$ component but there is not change in the monotonicity of the slope for all considered cases. Differences can be found for the counter-clockwise rotation of the handlebars where the monotonicity of $x$ component slope changes for all roll angles $\varphi$ while $y$ component always increases.

In Figure 8 we show the changes of $x$ and $y$ coordinate for flexible forks. The ranges of steering angle $\delta$ and roll angle $\varphi$ values are the same as for the stiff forks. During handlebar rotation there is not significant difference in $x$ component of front wheel's position. As a result the extreme values are almost the same. However, in the case of $y$ component we can see a difference close to zero value of the steering angle $\delta$, where we observe a multiple zero-crossing for all considered values of roll angle $\varphi$. For 

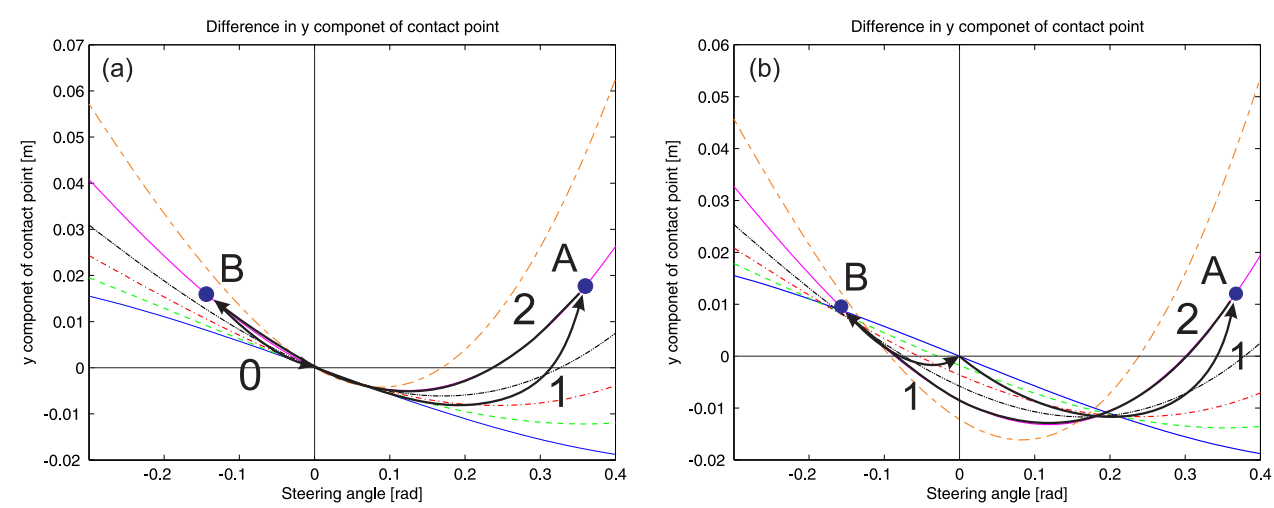

Fig. 9. Detailed view for change of front wheel tyre-ground contact position under changes in roll and steering angles for motorcycle with stiff and flexible forks. Colours of lines refer to: $\varphi=0 \mathrm{rad}$ - solid blue; $\varphi=0.18 \mathrm{rad}-$ dashed green; $\varphi=0.36 \mathrm{rad}$ - dash-dotted red; $\varphi=0.54 \mathrm{rad}-$ dash-double dotted black; $\varphi=0.72 \mathrm{rad}-$ three dash-dotted magenta $\varphi=$ $0.9 \mathrm{rad}$ - long and short dashed orange. Cornering maneuver is divided into three steps (three arrows). Two extreme values of steering angle $\delta$ are marked by blue dots (points A and B). By numbers next to arrows we indicate how many times the front tyre ground contact point crosses zero value of $y$ component in given stage of the maneuver (change of sign of $y$ component indicates crossing of the motorcycle plane of symmetry).

zero value of steering angle $\delta$ we observe the symmetric charts and exactly the same extreme values as in the case of rigid motorcycle. Changes can be seen in position of minimum because it changes in respect to the steering angle $\delta$ value, but it's nearly the same as for the rigid forks. The significant difference occurs for $\varphi=0.9 \mathrm{rad}$.

Detailed view of differences in $y$ component of the front tyre ground contact point during the maneuver for the stiff and the flexible forks are presented in Figures 9a and $9 \mathrm{~b}$ respectively.

As aforementioned, the maneuver is divided into three steps (three arrows). Two extreme values of steering angle $\delta$ are marked by blue dots (points A and B). By numbers next to arrows we indicate how many times the front tyre ground contact point crosses zero value of $y$ component in given stage of the maneuver (change of sign of $y$ component indicates crossing of the motorcycle plane of symmetry). It is clearly visible that in the case of rigid forks three crossings via zero occur as shown in Figure 9a. For front frame flexibility we observe different scenario as shown in Figure $9 \mathrm{~b}$. As it is easy to see the difference is in the left part of the diagram, hence to stage of maneuver where rider wants to go straight after exit a corner. In this case one can see that another zero crossing of $y$ component occurs. This comparison let us claim that one cannot ignore the flexibility of forks in the model of motorcycle because it has significant influence on the response of the system.

\subsection{Application possibilities}

Almost all racing motorcycles produced nowadays have the sophisticated electronic control units which provide not only information and feedback from the engine but also record the vast amount of vehicle's data during ride. Acquired data is used not only to measure the lap time but also to monitor other crucial parameters of the motorbike during the race. They are widely used to find the possible improvement of the bike's set-up. Finally the data are used as an input to real-time systems which help rider to get the maximum performance from the bike. One of those systems 
is the anti-wheelie which prevent looses of front tyre-ground contact [20]. Such subsystems can acquire data from the forks and shock positions to generate the adequate response from general engine control unit [21]. This cause the necessity to possessing reference value to compare it as fast as possible. Aforementioned algorithm includes this possibility and with extension mentioned in Section 3.2 is ideal for the real application. Nevertheless, to have fast and reliable algorithm we have to adjust its accuracy, i.e., the error value must be calculated with acceptable precision in reasonably short time. Decreasing accuracy by setting loop exit condition to one iteration gives result in average time $0.003 \mathrm{~s}$ and accuracy $0.0001 \mathrm{rad}$. Comparing those values with high-accuracy sensors available commercially let us claim that the accuracy is sufficient [22].

\section{Summary}

In this work we present how motorcycle's kinematic structure affects the capsize mode and appearances of vibrations in the motorcycle's plane of the symmetry. The presented results prove that the potential energy increases in tip over - capsize mode. We show the results of calculation of the pitch angle value for system with and without the lateral flexibility of the front forks. In both cases we reveal the same response when motorcycle is in the vertical position. However, even small change of the roll angle causes difference in response of system with finite lateral stiffness with comparison to the rigid one. In general for the stiff motorcycle with roll angle equal to zero, the clockwise rotation of the front wheel causes increase of the pitch angle value. When the value of the roll angle $\varphi$ increases we observe the maximum value of the pitch angle $\theta$ for smaller value of the steering angle $\delta$. In case of the counter-clockwise rotation changes in behaviour are more evident because initially increasing characteristic transforms into the decreasing one. For motorcycle with the lateral flexibility we observe the same behaviour, however the values of the pitch angle $\theta$ are larger in comparison to the stiff case. Moreover, the front forks flexibility results in moving of the zero crossing point. The lateral movement is also altered which reveals the calculation of the front tyre-ground contact point becomes complex. Changes in responses can be found in extreme values, amount and positions of zeros, steepness of the graph. Such analysis is vital for better design of the motorcycle chassis and gives first insight into motorcycle's dynamics behaviour. We claim that it is possible to apply presented algorithm to anti-wheelie system improving its precision and performance.

\section{Nomenclature}

Table 1. Nomenclature.

\begin{tabular}{ll}
\hline Parameter & Description \\
\hline$C_{1}$ & $\begin{array}{l}\text { Front wheel tyre-ground contact point/central point of the first global } \\
\text { system of coordinates }\end{array}$ \\
$C_{2}$ & $\begin{array}{l}\text { Rear wheel tyre-ground contact point/central point of the second global } \\
\text { system of coordinates }\end{array}$ \\
$W_{1}, W_{2}$ & $\begin{array}{l}\text { Front and rear wheel points laying at intersection of wheel axis and } \\
\text { wheel longitudinal plane of symmetry }\end{array}$ \\
$F$ & $\begin{array}{l}\text { Point at the steering head } \\
\text { Point at intersection of front upper frame longitudinal plane of } \\
\text { symmetry and front frame torsion axis }\end{array}$
\end{tabular}


Table 1. Continued.

\begin{tabular}{ll}
\hline$K$ & Point at the end of vertical part of front assembly \\
$\varphi$ & Roll angle of the motorcycle measured in respect to the rear frame \\
$\gamma$ & Roll angle of the front wheel \\
$\theta$ & Pitch angle of the rear frame \\
$\theta_{0}$ & Starting value of pitch angle - proper pitch angle in vertical position, \\
& 0.484 rad \\
$\theta_{f}$ & Pitch angle of the front frame \\
$\Delta \theta$ & Change of the pitch angle value \\
$\epsilon$ & Steering head angle \\
$\delta$ & Steering angle \\
$\delta^{\prime}$ & Steering angle of the front wheel measured on the ground \\
$\beta$ & Front frame torsion angle \\
$K_{f f}$ & Forks lateral stiffness \\
$C_{2} W_{2}$ & Rear wheel radius, $0.3 \mathrm{~m}$ \\
$C_{1} W_{1}$ & Front wheel radius $0.3 \mathrm{~m}$ \\
$W_{1} F$ & Rear frame length, $0.8 \mathrm{~m}$ \\
$F J$ & Upper front frame length, $0.2 \mathrm{~m}$ \\
$F I$ & Lower front frame length, $0.3 \mathrm{~m}$ \\
$I W_{1}$ & Front assembly offset, $0.1 \mathrm{~m}$ \\
\hline
\end{tabular}

Open Access This is an open access article distributed under the terms of the Creative Commons Attribution License (http://creativecommons.org/licenses/by/4.0), which permits unrestricted use, distribution, and reproduction in any medium, provided the original work is properly cited.

Publisher's Note The EPJ Publishers remain neutral with regard to jurisdictional claims in published maps and institutional affiliations.

\section{References}

1. H.B. Pacejka, Tyre and Vehicle Dynamics (Butterworth Heinemann, Oxford, U.K., 2002)

2. C. Koenen, The dynamic behaviour of motorcycles when running straight ahead and when cornering, Ph.D. dissertation, Delft Univ. Technol., 1983

3. R.S. Sharp, D.J.N. Limebeer, Multibody Syst. Dyn. 6, 123 (2001)

4. R.A. Lot, Meccanica 39, 207 (2004)

5. G. Franke, W. Suhr, F. Riess, Eur. J. Phys. 11, 116 (1990)

6. A. Saccon, J. Hauser, Veh. Syst. Dyn. 47, 221 (2009)

7. L. Leonelli, N. Mancinelli, Veh. Syst. Dyn. 53, 775 (2015)

8. Y. Miyamaru, G. Yamasaki, K. Aoki, Development of a motorcycle riding simulator, Soc. Auto. Eng. Jpn. 23, 121 (2002)

9. L. Nehaoua, S. Hima, H. Arioui, N. Seguy, S. Espie, Design and Modeling of a New Motorcycle Riding Simulator, American Control Conference USA, July 11-13, 2007

10. R. Sharp, C. Alstead, The influence of structural flexibilities on the straight-running stability of motorcycles, Veh. Syst. Dyn. 9, 327 (1980)

11. P. Spierings, The effects of lateral front fork flexibility on the vibrational modes of straight-running single-track vehicles, Veh. Syst. Dyn. 10, 21 (1981)

12. V. Cossalter, Motorcycle Dynamics (Lulu.com, 2006)

13. Society of Automotive Engineers "Vehicle dynamics terminology," in Proc. SAE, Warrendale, PA, 1976

14. J. Fajans, Steering in bicycles and motorcycles, Am. J. Phys. 68, 654 (2000) 
15. J. Craig, Introduction to Robotics: Mechanics and Control (Pearson/Prentice Hall, Boston, 2005)

16. S. Wolfram, The Mathematica Book (Wolfram Media, Incorporated 1996)

17. D.J.N. Limebeer, R.S Sharp, IEEE Control Syst. Mag. 26, 34 (2006)

18. V. Cossalter, R. Lot, A Motorcycle Multi-Body Model for Real Time Simulations Based on the Natural Coordinates Approach, Veh. Syst. Dyn. 3, 423 (2002)

19. S. Evangelou, D.J.N. Limebeer, Lisp programming of the Sharp 1971 motorcycle model, 2000, http://www.ee.ic.ac.uk/control/motorcycles

20. FIM World Championship Grand Prix Regulations, 2016

21. E. David, Everything You Wanted To Know About MotoGP's 2016 Unified Software, But Were Afraid To Ask, 2015, motomatters.com

22. SA-GY200/500V4-000 gyro datasheet, http://datrontechnology.co.uk/files/SAGYxxxV4-000-DINA4.pdf 